\title{
Concept Formation in Complex Domains
}

\author{
A. Ketterlin and J.J. Korczak \\ Centre de Recherche en Informatique - Université Louis Pasteur \\ 7, rue René Descartes, 67000 Strasbourg, France \\ e-mail: $\{$ alain, jjk\}edpt-info.u-strasbg.fr
}

\begin{abstract}
Most empirical learning algorithms describe objects as a list of attribute-value pairs. A flat attribute-value representation fails, however, to capture the internal structure of real objects. Mechanisms are therefore needed to represent the different levels of detail at which an object can be seen. A common structuring method is reviewed, and new principles of evaluation are proposed. As another way of enriching the representation language, a formalism is also proposed for multi-valued attributes, allowing the representation of sets of objects.
\end{abstract}

\section{Introduction}

Concept formation is the task of building a hierarchical organisation of concepts from a sequential presentation of unclassified objects. It is often called categorization, and has a central importance in many machine learning theories and applications. The basic CoBwEB algorithm, for instance, maintains a conceptual hierarchy by incrementally integrating new observations [1]. It performs a hillclimbing search through a space of conceptual hierarchies. The heuristic measure used, called category utility, is based on the average increase of predictivity for individual attributes. In fact, nodes in a conceptual hierarchy are labelled with a global predictivity score, which averages individual attributes' predictivity. This predictivity score, noted $\Pi(C)$, increases monotonically on any path from the root to a leaf, and quantifies a generalization partial ordering.

The aim of this work is to extend the space of representable observations for concept formation systems based on attribute-value representation formalism, since, it is largely restricted to low-level flat objects, i.e. objects with no internal structure. Complex domains require mechanisms to represent objects that can be viewed at different levels of detail [5]. In the case of the attribute-value representation formalism, values have to be allowed to be objects themselves, are observed and can be clustered on their own. In fact, little work has been done on the clustering of sets of objects. The easiest way to model sets is to allow multi-valued attributes. Such attributes take their value in a typed space of objects.

In both cases, embedded objects may be of any structural complexity. The fundamental assumption made throughout this paper is that they can be found in one single concept hierarchy. In essence, this means that objects of the same type can be compared and clustered, and leading us to a component-first strategy, since embedded objects must have been clustered for the englobing objects to be 
clustered. The reason for this restriction is that, in both cases, embedded objects are replaced by their conceptual signature (the concept that best describes it).

Section 2 introduces structured attributes. In Section 3, multi-valued attributes are described. In conclusion, the current position and some areas for future work are identified.

\section{Structured Attributes}

An important issue in representing complex objects is to be able to represent their internal structure. Real-world objects are often perceived as structured, i.e. decomposed into smaller, independent, embedded components. These are linked to the englobing object (the composite) by a PART-OF relation, the composite object being the aggregation of its components. The structure of instances can thus be described with a tree (a partonomy), where leaf-nodes represent primitive attributes (i.e. continuous or nominal) and internal nodes represent structured attributes, the root representing the object as a whole.

Values for structured attributes are objects. For any structured attribute, noted $A_{s}$, there exists a concept hierarchy, noted $\Gamma_{s}$, which partially orders the instance space, i.e. the space where values of $A_{s}$ reside. Suppose a concept $C$, covering the set of objects $\left\{O_{1}, \ldots, O_{N}\right\}$, the object $O_{n}$ taking value $O_{n s}$ for $A_{s}$. A predictivity measure for $A_{s}$ must evaluate the cohesiveness of the set $\left\{O_{1 s}, \ldots, O_{N s}\right\}$, which is the set of $A_{s}$ ' values in $C$. This evaluation is performed in three steps ${ }^{1}$ :

1. The embedded objects $O_{1 s}, \ldots, O_{N s}$ are located in the concept hierarchy $\Gamma_{s}$.

2. The most specific common ancestor, noted $\gamma_{g s}$, of the reached concepts is computed. According to the concept hierarchy $\Gamma_{s}$, this node is the most specific generalization of the set of embedded objects.

3. The predictivity score of $\gamma_{g s}$ is used as the predictivity score of $A_{s}$ in $C$.

The process of estimating predictivity for a structured attribute is equivalent to repeated applications of a climb-generalization-tree operator: the concept used to characterize a set of objects covers them all, and there are no more specific concepts having the same property.

At the object level, the representation of a structured attribute is an (embedded) object. At the concept level, structured attributes are represented with an embedded concept. The memory structure required to manipulate structured objects and concepts looks like a forest of conceptual hierarchies, where nodes of some trees point to nodes in other, more basic hierarchies: more precisely, it may be seen as a hierarchy of hierarchies.

The task of concept formation is defined as incremental unsupervised empirical learning. The mechanism for finding the conceptual representation of an

\footnotetext{
1 Thompson \& Langley's LABYRINTH system [5] is designed to deal with structured objects: their evaluation strategy also relies on the conceptual signature of embedded objects, but treats these as nominal values.
} 
attribute described above may be used in a totally incremental manner. When incorporating a new instance into a concept, the new most specific common ancestor of the covered values can be easily computed from its previous state and the conceptual signature of the incoming value. Moreover, seen values need not be kept, thus leaving memory space spare.

\section{Multi-Valued Attributes}

Multi-valued attributes allow the representation of properties whose values are sets of objects. Just as a structured attribute admits its own conceptual hierarchy, a multi-valued attribute $A_{m}$ is associated to a concept hierarchy $\Gamma_{m}$, where the elements of its values are clustered. Let us suppose a concept $C$, with an extensional definition of $N$ objects, each having a set $S_{n}=\left\{O_{n 1}, \ldots, O_{n m_{n}}\right\}$ as its value for $A_{m}$.

When examining the concept hierarchy $\Gamma_{m}$ according to the sets $S_{n}$, one can distinguish three kinds of nodes. Firstly, there may be nodes in $\Gamma_{m}$ that cover no object from any set $S_{n}$ : these are said to be empty, and are ignored. Secondly, there may be nodes that cover objects from only one set: these are said to be homogenous, and characterize the difference between a set and all the others. The third type of node, the most informative, is called a heterogenous, or mixed, node $^{2}$. It is always possible to find nodes in $\Gamma_{m}$ that cover objects from at least two distinct sets $S_{i}$ and $S_{j}$.

Intuitively, a predictive characterization of a set of sets of objects relies on highly predictive (specific) mixed nodes. Since a mixed node's parent is also mixed, a good characterization of a set of multiple values can be found at the interface between mixed and homogenous nodes in $\Gamma_{m}$. This means that the more informative mixed nodes are the most specific ones. On the other hand, informative homogenous nodes are the most general ones. This leads us to the definition of central nodes. Central nodes are mixed nodes with no mixed subnode, i.e. the most specific mixed nodes. The set of central nodes in a concept hierarchy $\Gamma_{m}$, according to the set of values $\left\{S_{1}, \ldots, S_{N}\right\}$, forms a generalization of these values. Moreover, predictivity scores of central nodes give an evaluation of the predictivity of this generalization. All central nodes do not, however, cover the same proportion of sets, nor the same proportion of objects. It seems intuitive to weight contributions of central nodes according to these factors. The exact formula to compute $A_{m}$ 's predictivity is:

$$
\sum_{l=1}^{L} \frac{n_{e}\left(S, \gamma_{l}\right)}{N} \cdot \frac{n_{o}\left(S, \gamma_{l}\right)}{N_{o}} \cdot \Pi\left(\gamma_{l}\right)
$$

where $L$ is the number of central nodes (noted $\gamma_{1}, \ldots, \gamma_{L}$ ), $S$ is the set of values $\left\{S_{1}, \ldots, S_{N}\right\}, n_{e}\left(S, \gamma_{l}\right)$ is the number of sets in $S$ having a non-empty intersection with the extensionnal definition of $\gamma_{1}, n_{o}\left(S, \gamma_{l}\right)$ is the total number of objects from $S_{1}, \ldots, S_{N}$ covered by $\gamma_{l}$, and $N_{o}=\sum_{n=1}^{N}\left|S_{n}\right|$.

\footnotetext{
${ }^{2}$ The formal definitions of the empty, homogenous and mixed node can be found in [3].
} 
In the case of a structured attribute $A_{s}$, the conceptual representation of $A_{s}$ was a single concept from $\Gamma_{s}$. For a multi-valued attribute $A_{m}$, the conceptual representation of $A_{m}$ is a set of concepts from $\Gamma_{m}$. Thus, a concept described with multi-valued attributes points to several other, more basic, concepts.

\section{Conclusions}

The motivation for these new types of attributes is to extend the concept representation formalism. They do not entail any modification of the original control structure of COBWEB, nor do they fundamentally change its evaluation function. Structured and multi-valued attributes may be seen as type-constructors, and can be contrasted with continuous and nominal attributes, which are called primitive attributes. Measures of predictivity for primitive attributes are part of the background knowledge. In contrast, in the case of structured and multi-valued attributes, predictivity measures are based on self-made concept hierarchies. The two type-constructor attributes allow concept formation to take place in many domains [3]. In particular, several data models use the same mechanisms to represent complex data [2].

Another aspect of structured concepts is their relation to abstraction, as defined in [4]. In this framework, abstraction is a knowledge transmutation that decreases the level of detail at which objects are observed. This was exactly the motivation for structured objects, i.e. to capture the different levels of detail. Knowledge transmutations like concretion would be, in our framework, to split a structured attribute into its descendants in the partonomy. In contrast, abstraction, would be to merge nodes of the partonomy to form a new, intermediate, structured attribute. Such transformations of the partonomy carry out some form of constructive induction. Moreover, heuristics to decide when to perform abstraction or concretion are available, through examination of relative predictivity variations at different levels in the partonomy. The result would be a concept formation system that, at the same time, learns the best representation for objects.

\section{References}

1. Fisher, D.H. (1987). Knowledge Acquisition via Incremental Conceptual Clustering. Machine Learning, 2, pp 139-172.

2. Hull, R., \& King, R. (1987). Semantic Database Modeling: Survey, Applications, and Research Issues. ACM Computing Surveys, 19, pp 201-260.

3. Ketterlin, A., \& Korczak, J.J. (1993). Concept Formation in Complex Domains: Structured and Multi-Valued Attributes. Research Report, Centre de Recherche en Informatique, Université Louis Pasteur, Strasbourg.

4. Michalski, R.S. (1993). Inferential Theory of Learning as a Conceptual Basis for Multistrategy Learning. Machine Learning, 11, pp 111-151.

5. Thompson, K., \& Langley, P. (1991). Concept Formation in Structured Domains. In D.H. Fisher, M.J. Pazzani, \& P. Langley (Eds.), Concept Formation: Knowledge and Experience in Unsupervised Learning, pp 127-161. Morgan Kaufmann. 